\title{
(6) OPEN ACCESS \\ Treatment of Advanced Glaucoma Study: a multicentre randomised controlled trial comparing primary medical treatment with primary trabeculectomy for people with newly diagnosed advanced glaucoma—study protocol
}

\author{
Anthony J King, ${ }^{1}$ Gordon Fernie, ${ }^{2}$ Augusto Azuara-Blanco, ${ }^{3}$ Jennifer M Burr, ${ }^{4}$ \\ Ted Garway-Heath, ${ }^{5,6}$ John M Sparrow, ${ }^{7}$ Luke Vale, Jemma Hudson, ${ }^{2}$ \\ Graeme MacLennan, ${ }^{2}$ Alison McDonald, ${ }^{2}$ Keith Barton, ${ }^{6}$ John Norrie ${ }^{2}$
}

'Department of Ophthalmology, Nottingham University Hospitals NHS Trust, Nottingham, UK ${ }^{2}$ Centre for Healthcare Randomised Trials (CHaRT), Health Services Research Unit, University of Aberdeen, Health Services Research Unit, University of Aberdeen, Aberdeen, UK

${ }^{3}$ Centre for Public Health, Queen's University Belfast Royal Victoria Hospital, Belfast, Northern Ireland, UK

${ }^{4}$ School of Medicine, University of St Andrews, St Andrews, Fife, UK

${ }^{5}$ Institute of Ophthalmology, University College London, London, UK

${ }^{6}$ Moorfields Eye Hospital, NHS Foundation Trust, London, UK ${ }^{7}$ Department of Ophthalmology, Bristol Eye Hospital, Bristol, UK ${ }^{8}$ Institute of Health and Society, Newcastle University, Newcastle, UK

Correspondence to Anthony J King, Department of Ophthalmology, Nottingham University Hospitals NHS Trust, Nottingham NG5 1PB, UK; anthony.king@nottingham. ac.uk

Received 21 June 2017 Revised 15 September 2017 Accepted 23 September 2017 Published Online First 26 October 2017

Check for updates

To cite: King AJ, Fernie $\mathrm{G}$,

Azuara-Blanco A, et al.

Br J Ophthalmol

2018:102:922-928.

\section{ABSTRACT}

Background Presentation with advanced glaucoma is the major risk factor for lifetime blindness. Effective intervention at diagnosis is expected to minimise risk of further visual loss in this group of patients.

Aim To compare clinical and cost-effectiveness of primary medical management compared with primary surgery for people presenting with advanced open-angle glaucoma (OAG).

Methods Design: A prospective, pragmatic multicentre randomised controlled trial (RCT).

Setting Twenty-seven UK hospital eye services.

Participants Four hundred and forty patients presenting with advanced $O A G$, according to the Hodapp-Parish-Anderson classification of visual field loss.

Intervention Participants will be randomised to medical treatment or augmented trabeculectomy (1:1 allocation minimised by centre and presence of advanced disease in both eyes)

Main outcome measures The primary outcome is vision-related quality of life measured by the National Eye Institute-Visual Function Questionnaire-25 at 24 months. Secondary outcomes include generic EQ-5D-5L, Health Utility Index-3 and glaucomarelated health status (Glaucoma Utility Index), patient experience, visual field measured by mean deviation value, logarithm of the mean angle of resolution visual acuity, intraocular pressure, adverse events, standards for driving and eligibility for blind certification. Incremental cost per quality-adjusted life-year (QALY) based on EQ-5D-5L and glaucoma profile instrument will be estimated.

Results The study will report the comparative effectiveness and cost-effectiveness of medical treatment against augmented trabeculectomy in patients presenting with advanced glaucoma in terms of patient-reported health and visual function, clinical outcomes and incremental cost per QALY at 2 years.

Conclusions Treatment of Advanced Glaucoma Study will be the first RCT reporting outcomes from the perspective of those with advanced glaucoma.

Trial registration number ISRCTN56878850, Preresults.

\section{INTRODUCTION}

Advanced visual field (VF) loss at glaucoma diagnosis is the major risk factor for lifetime blindness. Reducing intraocular pressure (IOP) by medications, surgery or laser is the only currently available treatment option for glaucoma. ${ }^{2-9}$ Glaucoma guidelines suggest that primary surgery may be a suitable option for presentation with advanced glaucoma. ${ }^{10-12}$

Evidence from a systematic review, ${ }^{2}$ including four randomised controlled trials (RCTs), ${ }^{3}$ 13-15 comparing primary medical versus surgical treatment for open-angle glaucoma (OAG) suggests that treatment failure was more likely when the primary treatment was medication (OR 3.90, 95\% CI 1.60 to 9.53 ; HR $7.27,95 \%$ CI 2.23 to 25.71 ). Data from the largest and most recent of these RCTs found no substantial difference in patient-reported outcomes between intervention groups at 5 years. ${ }^{16}$ However, this study did not include people with advanced glaucoma. Subsequent to these studies being completed, many more glaucoma medications have become available with better efficacy in terms of IOP lowering and less side effects. ${ }^{17}$ Trabeculectomy remains the conventional primary surgery, but the technique has evolved ${ }^{1819}$ to include the use of wide application mitomycin C (MMC), ${ }^{20}$ releasable sutures $^{21}$ and extensive postoperative manipulation $^{22}$ to improve outcomes. ${ }^{23}$

Given that advanced glaucoma is a sight-threatening disease with uncertainty about the best primary treatment option, there is a need for a highquality study comparing contemporary medications with primary trabeculectomy for newly diagnosed advanced glaucoma reporting outcomes relevant to patients, clinicians and health services.

\section{PATIENTS AND METHODS}

The Treatment of Advanced Glaucoma Study (TAGS) is a pragmatic ${ }^{24} 25$ multicentre RCT comparing primary medical treatment with primary augmented trabeculectomy with the primary outcome assessment at 2 years post randomisation. Participants will be randomised to medical treatment or augmented trabeculectomy (1:1 allocation 


\section{Box Inclusion and exclusion criteria}

\section{Inclusion criteria}

1. Severe glaucomatous visual field loss (Hodapp classification) in one or both eyes at presentation on any of these criteria:

a. Mean deviation $<-12.00 \mathrm{~dB}$.

b. $>50 \%$ of points defective in the pattern deviation probability plot at the $5 \%$ level (>27 points on $24-2$ HVF).

c. $>20$ points defective at the $1 \%$ level.

d. A point in the central 5 degrees has a sensitivity of $0 \mathrm{~dB}$.

e. Points within 5 degrees of fixation $<15 \mathrm{~dB}$ sensitivity in both upper and lower hemifields.

2. Diagnosis of open-angle glaucoma including pigment dispersion glaucoma, pseudo-exfoliative glaucoma and normal tension glaucoma.

3. Adult $\geq 18$ years.

4. Ability to provide informed consent and willingness to participate in the trial.

Exclusion criteria

1. Visual field defects not meeting advanced visual field loss criteria in either eye.

2. Primary angle closure glaucoma and all other secondary glaucomas.

3. Inability to undergo incisional surgery due to inability to lie flat or unsuitable for anaesthetic.

4. High risk of trabeculectomy failure such as previous conjunctival surgery, complicated cataract surgery.

5. Women who are

a. (i) pregnant, (ii) nursing, (iii) planning a pregnancy and (iv) of childbearing potential not using a reliable method of contraception.

minimised by centre and presence of advanced disease in both eyes). The trial's protocol reflects routine care to ensure that the results are representative of current clinical practice.

\section{Participants}

Adults ( $\geq 18$ years) presenting with advanced (severe) glaucoma in at least one eye will be invited to participate. Advanced disease is classified according to the 'severe' category of VF loss using the Hodapp classification of glaucoma severity ${ }^{26}$ (see box for a full list of eligibility criteria). Two baseline Humphrey VFs (24-2 SITA Standard) will be performed on all participants prior to randomisation to confirm eligibility. If both eyes are eligible, it is the participant, not the eye, who is randomised to treatment; both eyes receive the same intervention.

Participants will be recruited from an estimated 27 hospitals in the UK over a 36-month period. Participants will be followed for 2 years from randomisation (see table 1 for the schedule of assessments).

\section{Study interventions}

An individualised target IOP will be set at baseline according to the algorithm developed by the Canadian Consensus on target IOP setting. ${ }^{27}$

In the primary medical treatment arm, participants will start on one or more medications at their initial visit depending on the judgement of the treating clinician and as advised by the National Institute for Health and Care Excellence (NICE) glaucoma guideline, ${ }^{10}$ with subsequent additional medication based on clinician judgement. All currently available licensed drops may be used. If drops fail to lower the IOP adequately, oral carbonic anhydrase inhibitors may be used. If medical treatment fails, patients will be offered glaucoma surgery.

In the primary trabeculectomy group, surgery will be undertaken within 3 months of randomisation by a surgeon who specialises in glaucoma or a glaucoma fellow who has performed at least 30 trabeculectomies. Patients' IOP will be medically controlled until glaucoma surgery is undertaken. In trabeculectomy augmented with MMC, a 'guarded fistula' is created by making a small hole in the eye, covered by a flap of partial thickness sclera. ${ }^{1828}$ After glaucoma surgery, medical treatment may be introduced if the IOP is above the desired target.

Table 1 Timing of outcome measurements

\begin{tabular}{|c|c|c|c|c|c|c|c|c|}
\hline & \multirow[b]{2}{*}{ Baseline } & \multicolumn{7}{|c|}{ Post randomisation (months) } \\
\hline & & 1 & 3 & 4 & 6 & 12 & 18 & 24 \\
\hline \multicolumn{9}{|l|}{ Clinical } \\
\hline Medical history & $x$ & & & & & & & \\
\hline Humphrey visual field mean deviation & $\mathrm{x}$ & & & $\mathrm{x}$ & & $\mathrm{x}$ & & $\mathrm{x}$ \\
\hline Esterman Visual Field & $x$ & & & & & & & $x$ \\
\hline LogMAR visual acuity & $\mathrm{x}$ & & & $\mathrm{x}$ & & $\mathrm{x}$ & & $\mathrm{x}$ \\
\hline Intraocular pressure & $x$ & & & $\mathrm{x}$ & & $x$ & & $x$ \\
\hline Standard clinical examination & $\mathrm{x}$ & & & & & $\mathrm{x}$ & & $x$ \\
\hline \multicolumn{9}{|l|}{ Patient experience } \\
\hline NEI-VFQ-25 & $\mathrm{x}$ & & & $\mathrm{x}$ & & $\mathrm{x}$ & & $\mathrm{x}$ \\
\hline EQ-5D-5L* & $x$ & $x$ & $\mathrm{x}$ & & $x$ & $\mathrm{x}$ & $\mathrm{x}$ & $\mathrm{x}$ \\
\hline HUI-3* & $x$ & $\mathrm{x}$ & $x$ & & $x$ & $x$ & $x$ & $\mathrm{x}$ \\
\hline $\mathrm{GUI}^{*}$ & $x$ & $x$ & $\mathrm{x}$ & & $\mathrm{x}$ & $x$ & $\mathrm{x}$ & $\mathrm{x}$ \\
\hline Patient experience questions & $\mathrm{x}$ & $\mathrm{x}$ & $\mathrm{x}$ & & $x$ & $\mathrm{x}$ & $\mathrm{x}$ & $\mathrm{x}$ \\
\hline \multicolumn{9}{|l|}{ Health economics } \\
\hline Healthcare utilisation (including hospital visits) & & & & $\mathrm{x}$ & & $\mathrm{x}$ & & $\mathrm{x}$ \\
\hline Participant cost & & & & $\mathrm{x}$ & & $x$ & & $\mathrm{x}$ \\
\hline Participant time and travel & & & & & & & $\mathrm{x}$ & \\
\hline
\end{tabular}

*Additional questionnaire undertaken immediately prior to trabeculectomy surgery; discrete choice experiment at 27 months;

GUI, Glaucoma Utility Index; HUI-3, Health Utility Index; LogMAR, logarithm of the mean angle of resolution; NEI-VFQ25, National Eye Institute Visual Function Questionnaire (25 items). 


\begin{tabular}{|c|c|c|}
\hline & Outcome & Analysis \\
\hline $\begin{array}{l}\text { Primary } \\
\text { objective }\end{array}$ & $\begin{array}{l}\text { Patient-centred: } \\
\text { Vision-specific health profile (NEI-VFQ25) at } \\
24 \text { months }\end{array}$ & Intention to treat. \\
\hline $\begin{array}{l}\text { Secondary } \\
\text { objectives }\end{array}$ & $\begin{array}{l}\text { Patient-centred: } \\
\text { Patient-reported health status, HUI-3; EQ-5D-5L } \\
\text { (5-level), GUI, NEI-VFQ25; patient experience. } \\
\text { Clinical: } \\
\text { Visual field mean deviation at } 24 \text { months. } \\
\text { Intraocular pressure; logMAR visual acuity; need } \\
\text { for cataract surgery; visual standards for driving; } \\
\text { registered visual impairment; safety. } \\
\text { Economic: } \\
\text { Incremental cost per QALY gained (based on } \\
\text { responses to the EQ-5D-5L; HUI-3); incremental } \\
\text { cost per QALY gained (based on responses to } \\
\text { glaucoma profile instrument (GPI)); incremental } \\
\text { costs to the NHS, personal social services and } \\
\text { patients. }\end{array}$ & $\begin{array}{l}\text { Profile over time } \\
\text { will be analysed by } \\
\text { repeated measures } \\
\text { using a linear mixed } \\
\text { model. Subgroup } \\
\text { analyses will explore } \\
\text { potential effect } \\
\text { modification of gender, } \\
\text { age, one or both eyes } \\
\text { affected and extent } \\
\text { of visual field loss at } \\
\text { baseline (<-20 dB, } \\
\geq 20 \mathrm{~dB} \text { ) on the primary } \\
\text { outcomes. }\end{array}$ \\
\hline
\end{tabular}

GPI, glaucoma profile instrument; GUI, Glaucoma Utility Index; HUI-3, Health Utility Index; LogMAR, logarithm of the mean angle of resolution; NEI-VFQ25, National Eye Institute Visual Function Questionnaire (25 items); NHS, National Health Service; QALY, quality-adjusted life-year.

Where both eyes are eligible and the participant is allocated to the surgery arm, a decision as to which eye will undergo trabeculectomy first will be made by the clinician and patient together. A period of 2-3 months will be allowed between these operations.

\section{Summary of outcomes}

The primary outcome is vision-related quality of life (QoL) collected with the National Eye Institute Visual Function Questionnaire (25 items) (NEI-VFQ25). Secondary outcomes include clinical outcomes: mean deviation (MD) on Humphrey VF testing, logarithm of the mean angle of resolution (logMAR) visual acuity, IOP, complications of treatment, need for cataract surgery; patient-reported outcomes: generic health status (EQ-5D-5L and Health Utility Index (HUI-3)), glaucoma health status (glaucoma profile instrument (GPI)), patient experience; and health economic outcomes: quality-adjusted life-year (QALYs) and incremental cost per QALY (tables 1 and 2).

The NEI-VFQ25 is a vision-specific patient-reported QoL instrument widely used to evaluate visual outcomes in glaucoma. ${ }^{29-32}$ Generic EQ-5D-5L, ${ }^{33}$ HUI- $3^{34}$ and the glaucoma-specific, $\mathrm{GPI}^{35}$ will be collected to generate utility outcomes. VF MD change measures the amount of vision loss due to glaucoma. $\mathrm{VF}$ damage is the major clinical measure of the functional impact of glaucoma, which adversely influences QoL. ${ }^{29}{ }^{36-38}$ VFs eligible for analysis will have to achieve a predefined reliability criterion (false positives $<15 \%$ ). VFs will be assessed by an independent VF reading centre, masked to the treatment received by the study participant. The independent reading centre will evaluate MD change and whether the Esterman Visual Fields achieve driving standard levels.

IOP will be measured by Goldmann tonometry in $\mathrm{mm} \mathrm{Hg}$. The measurement will be undertaken by two observers, the first observer making the measurement and the second reading it from the measurement dial. Two measurements will be taken and a third if there is a discrepancy $>3 \mathrm{~mm} \mathrm{Hg}$ between the first two. The mean of these values will be used.

Best-corrected $\log$ MAR visual acuity at $4 \mathrm{~m}$ will be measured on each eye and binocularly. Complications of surgery, need for cataract surgery and therapy changes, will be captured from the participants' case records. All clinical outcomes will be recorded on a trial-specific case report form (CRF). If a participant is eligible to be registered as visually impaired or severely visually impaired in the opinion of the consulting clinician, this will be recorded in the study CRF at 24 months.

\section{Economic outcomes}

Costs of treatments (surgery/medications) including time in hospital and secondary care use will be based on data collected in the trial CRFs. Primary care, personal social service use and participant costs will be collected via questionnaires at 4, 12 and 24 months post randomisation. Responses to the EQ-5D-5L (recommended for use by NICE in the UK), HUI-3 (which has vision-related questions) and GPI (which should be more sensitive to changes in glaucoma) will be combined with the relevant scoring tariffs ${ }^{35} 3940$ to produce QALYs. Glaucoma-specific scoring tariffs will be elicited in this study using a discrete choice experiment, replicating the method adopted by Burr et $a l^{35}$ with a sample of people with advanced glaucoma. Costs and QALYs will be combined in a cost-utility analysis both 'within trial' and modelled over the patient's lifetime; with the model informed by previous models conducted. ${ }^{41-43}$

\section{Sample size}

The primary outcome is patient-reported vision-related QoL measured by the NEI-VFQ25 at 24 months. A study with 190 participants in each group will have $90 \%$ power at $5 \%$ significance level to detect a difference in means of 0.33 of an SD; this translates to 6 points on the NEI-VFQ25 assuming a common SD of 18 points from previous work in patients with advanced glaucoma. ${ }^{44}$ Seven points is a likely minimally important difference based on our pilot work on NEI-VFQ25 scores in patients with glaucoma, ${ }^{45}$ but there is uncertainty and so we have opted for a more conservative six-point difference, which is supported by the literature for another chronic eye disease, macular degeneration. ${ }^{46}$ Assuming a $13.5 \%$ dropout due to death and participants declining follow-up, we need to randomise 440 participants to detect this difference.

For the secondary clinical outcome (VF MD), the study has $90 \%$ power at a $5 \%$ level of significance to detect a $1.3 \mathrm{~dB}$ difference in MD between groups after 2 years. This was derived from a subgroup of patients with advanced glaucoma ${ }^{247}$ and is a clinically meaningful difference in the context of advanced glaucoma, given that progressive loss tends to be linear, ${ }^{48} 49$ with small changes over a short period extrapolating to large changes over a patient's lifetime.

\section{Recruitment}

Patients likely to be eligible for the trial will be identified at the initial consultation for glaucoma and provided with a participant information leaflet. Eligible participants, who agree to take part, will sign a consent form before being randomised. Recruitment will be performed by the ophthalmologist and must be completed within 3 months of glaucoma diagnosis.

Randomisation will be performed at recruitment centres using the remote-automated computer randomisation service at the Centre for Healthcare Randomised Trials, either over the internet or by telephone. Randomisation will be minimised by centre and whether there is advanced glaucoma in both eyes. The unit of randomisation is the participant (not the eye). 


\section{Adverse events/safety reporting}

Serious adverse events (SAEs) related to participants' glaucoma care or participation in the trial will be reported in accordance with the guidance from the UK Health Research Authority (http://www.hra.nhs.uk/research-community/duringyour-research-project/safety-reporting/). Table 3 describes the expected adverse events.

All related SAEs will be summarised and reported to the Ethics Committee, the funder and the Trial Steering Committee in regular progress reports. Any serious, related and unexpected events will be expeditiously reported (no later than 15 calendar days after the trial team are first aware of the event). ${ }^{50}$

\section{Procedure}

Table 1 displays the timing of the trial's outcome measurements. At baseline, following consent but prior to randomisation, participants' relevant medical history, IOP, Humphrey visual fields and best-corrected $\log$ MAR visual acuity will be measured. A general ophthalmic examination including central corneal thickness will also be undertaken. Participants will complete a questionnaire including the NEI-VFQ25, EQ-5D-5L, HUI-3, GPI and a question asking about the patient's experience of glaucoma.

Most of the outcomes will be gathered at four time points across the 2-year follow-up as illustrated in figure 1 (see table 1 for details).

\section{Statistical analyses}

Baseline characteristics, follow-up measurements and safety data will be described using the appropriate descriptive summary measures. The primary outcome, NEI-VFQ25 score, will be analysed using linear regression adjusting for baseline NEI-VFQ25 score and other prognostic variables, for example, amount of vision loss and IOP at baseline, one or both eyes affected by advanced glaucoma. We will also explore the profile of NEI-VFQ25 score over time using a linear mixed model to analyse data from all time points. All models will include a random effect for surgeon. The primary analysis strategy will be intention to treat. We will describe the amount and patterns of missing data and use appropriate methods,${ }^{51}$ if required, to run sensitivity analysis to test assumptions. ${ }^{52}$

There is potential for cross-over to the alternative treatment (non-compliance with allocation). In addition to the 'effectiveness' estimate from the intention-to-treat analyses, we will explore 'efficacy' estimates using causal modelling methods suitable for complex interventions, ${ }^{53}$ if required.
Secondary outcomes will be analysed with a similar strategy, with models suitable for the outcome (ie, logistic regression for the dichotomous outcome 'need for cataract surgery' at 2 years). Outcomes measured at the eye level will be analysed initially using data from the index eye only. For participants with both eyes eligible, the eye with the better MD value (less severe VF damage) is nominated the index eye for the purposes of the statistical analyses.

Sensitivity analysis using data from all eligible eyes will be done, including a random effect at the participant level to reflect the lack of independence of eyes within participants. All treatment effects will be derived from these models and presented with $95 \%$ CIs.

Planned subgroup analyses are intended to explore potential effect modifications of gender, age, one or both eyes affected and extent of VF loss in the index eye at baseline $(<-20 \mathrm{~dB}$, $\geq 20 \mathrm{~dB}$ ) on the primary outcomes. Subgroup by treatment interaction will be assessed by including interaction terms in the models outlined above.

The Data Monitoring Committee will monitor safety and other data at 6-monthly intervals during the recruitment phase of the trial. Due to the staggered nature of recruitment and the primary outcome measurement at 2 years, we do not anticipate early termination for benefit. We have planned for one main effectiveness analysis at the end of the trial. All statistical analysis will be detailed in the Statistical Analysis Plan, which will be completed before the final analysis is started.

The study adheres to the tenets of the Declaration of Helsinki and the principles of Good Clinical Practise (GCP), and is in accordance with all applicable regulatory guidance, including, but not limited to, the Research Governance Framework. TAGS' protocol and patient-facing documentation were prospectively reviewed and approved by the Derby 1 Research Ethics Committee (Ref Number 13/EM/00395). Local NHS Research and Development (R\&D) approvals were obtained prior to commencement of the trial at the participating sites. An independent data and safety monitoring committee oversees the trial.

\section{Funding}

The trial is funded by a grant awarded by the Health Technology Assessment (NIHR HTA) programme (project number 12/35/38). TAGS is registered on the ISRCTN registry: 56878850 .

Nottingham University Hospitals NHS Trust sponsors the trial and provides the necessary trial insurance.

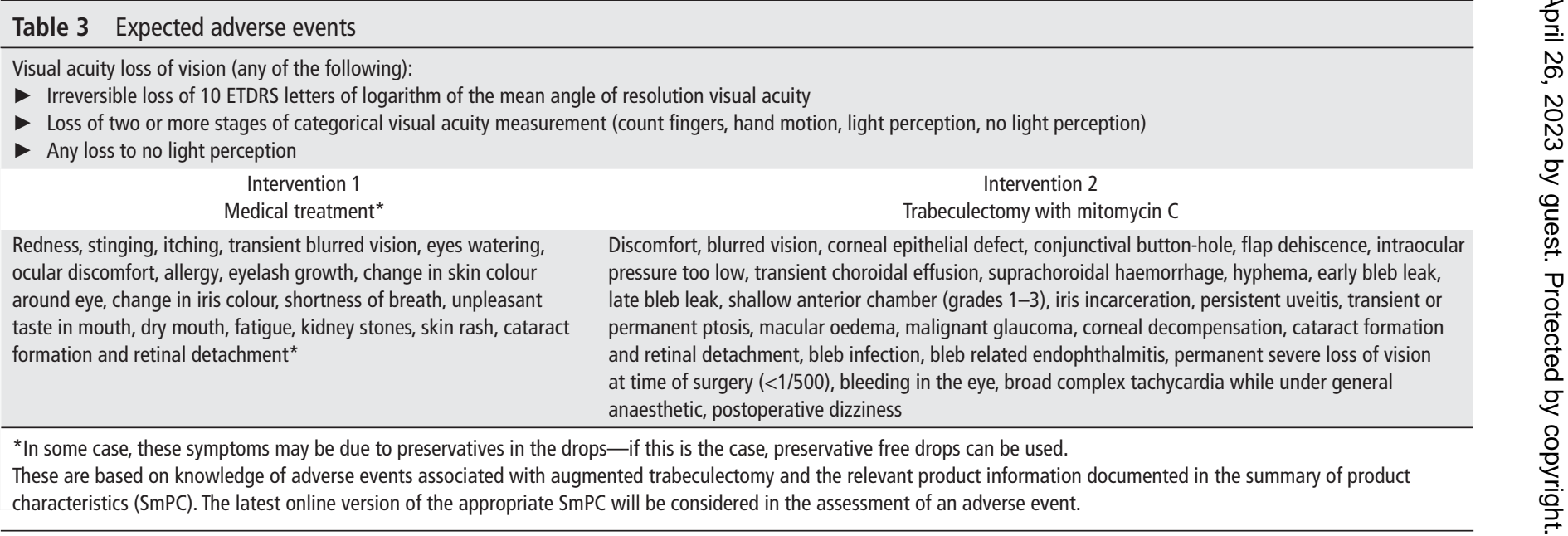



Flow diagram: Treatment of Advanced Glaucoma Study (TAGS): A RCT comparing primary medical
treatment with primary augmented trabeculectomy

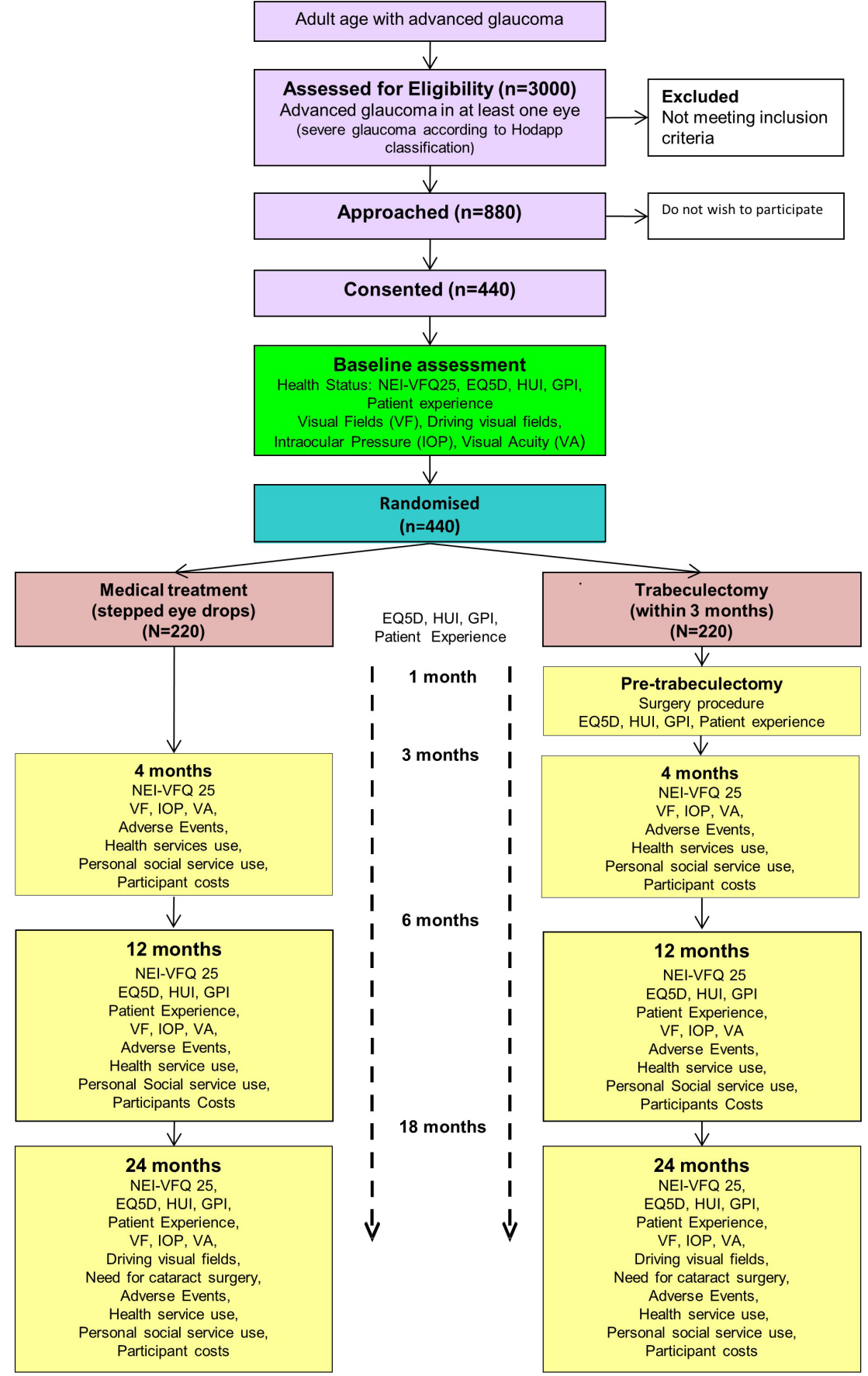

Figure 1 Study flow chart with outcome timeline. GPI, glaucoma profile instrument; HUI, Health Utility Index; NEI-VFQ25, National Eye Institute Visual Function Questionnaire (25 items); RCT, randomised controlled trial.

\section{DISCUSSION}

Major changes in the efficacy, safety and variety of glaucoma drops available over the last two decades as well as significant modifications to trabeculectomy surgery have improved safety and efficacy outcomes. ${ }^{18} 23$

There is uncertainty regarding the optimal primary treatment pathway for people presenting with advanced glaucoma. There is a recognition that advanced glaucoma at presentation needs to be treated more aggressively. ${ }^{10-12}$ In the UK, only about $30 \%$ of UK ophthalmologists follow the NICE guidelines for primary surgery. ${ }^{54}$ Clinicians indicated that robust evidence supporting the best primary approach would change their practice. ${ }^{54}$ The TAGS study aims to address this evidence gap.

Patient-reported outcomes are an important component of treatment choice and are related to the extent of VF loss. ${ }^{50} 55$ TAGS is the first glaucoma surgery RCT with patient-reported vision-related QoL as the prespecified primary outcome. As both treatments being tested have proven efficacy in treating 
glaucoma, there may be little measurable difference in clinical outcomes. Loss of visual function leads to disability in tasks of daily living. ${ }^{56-64}$ In patients with advanced glaucoma, because of their limited visual reserve, further VF loss is likely to result in noticeable difference in visual function in all aspects of life related to vision.

The participant 'journey' through both treatment options following diagnosis will be recorded with a vision-specific (NEIVFQ25), generic (EQ-5D-5L and HUI-3) and glaucoma-specific (GPI) patient-reported questionnaires capturing any QoL differences between a primary surgery and a primary medication care pathway. We will also explore which generic health status questionnaires, one with a specific visual function domain (HUI-3) and one without (EQ-5D-5L), is sensitive to any differences between primary treatment options.

Generic QoL assessments have not been previously undertaken in a medicine versus surgery trial for glaucoma, although the Collaborative Initial Glaucoma Treatment Study ${ }^{31}$ reported on differences in local eye symptoms and visual activities related to interventions. ${ }^{16}$

Measuring our outcome at 2 years will allow us to track the patient journey through the active initial management period for their glaucoma and will provide us with meaningful information regarding patient-reported outcomes, clinical and safety outcomes and economic outcomes of the two treatment options tested. However, glaucoma is a slowly progressive condition and it may take many years for differences in the outcomes of treatment choices to be revealed. ${ }^{47}{ }^{65-67}$ Patients with glaucoma live for many years following their diagnosis ${ }^{68-72}$ and it is therefore essential to obtain further information about the effect of treatment options in the medium to long term to better inform our patients of lifetime outcomes with different treatment options and allow more effective economic modelling. We therefore plan to seek further funding to allow further evaluation of this cohort at 5 years.

Contributors $A K$ leads the trial and is the chief investigator. $A A B, J B, T G H, J S$, LV, GM, AM, KB and JN are grant-holders and developed the protocol. GF is trial manager and revised the protocol. JH and GM wrote the statistical analysis plan. All authors contributed to this article.

Funding Nottingham University Hospitals NHS Trust sponsors the trial and provides the necessary trial insurance. The trial is funded by a grant awarded by the Health Technology Assessment (NIHR HTA) programme (project number 12/35/38).

Disclaimer The views expressed are those of the authors and not necessarily those of the NHS, the NIHR or the Department of Health.

Competing interests TAGS is funded by the National Institute for Health Research Health Technology Assessment (NIHR HTA) programme (project number 12/35/38). JMB, GM, AM and JMS report grants from NIHR Health Technology Assessment Programme during the conduct of the study. LV reports grants from NIHR HTA Programme during the conduct of the study; and membership of the NIHR Health Technology Assessment Programme and a director of the NIHR Research Design Service. JN reports grants from NIHR HTA during the conduct of the study; personal fees from NIHR Editors Board, other from NIHR HTA General Board, outside the submitted work.

Ethics approval The study adheres to the tenets of the Declaration of Helsinki and the principles of Good Clinical Practice (GCP), and is in accordance with all applicable regulatory guidance, including, but not limited to, the Research Governance Framework. TAGS' protocol and patient-facing documentation were prospectively reviewed and approved by the Derby 1 Research Ethics Committee(ref number 13/EM/00395). Local NHS Research and Development (R\&D)approvals were obtained prior to commencement of the trial at the participating sites. An independent data and safety monitoring committee oversees the trial.

Provenance and peer review Not commissioned; externally peer reviewed.

Open access This is an open access article distributed in accordance with the terms of the Creative Commons Attribution (CC BY 4.0) license, which permits others to distribute, remix, adapt and build upon this work, for commercial use, provided the original work is properly cited. See: http://creativecommons.org/ licenses/by/4.0/

(C) Article author(s) (or their employer(s) unless otherwise stated in the text of the article) 2018. All rights reserved. No commercial use is permitted unless otherwise expressly granted.

\section{REFERENCES}

1 Peters D, Bengtsson B, Heijl A. Factors associated with lifetime risk of open-angle glaucoma blindness. Acta Ophthalmol 2014:92:421-5.

2 Burr J, Azuara-Blanco A, Avenell A, et al. Medical versus surgical interventions for open angle glaucoma. Cochrane Database Syst Rev 2012:9:CD004399.

3 Lichter PR, Musch DC, Gillespie BW, et al. Interim clinical outcomes in the Collaborative Initial Glaucoma Treatment Study comparing initial treatment randomized to medications or surgery. Ophthalmology 2001;108:1943-53.

4 Comparison of glaucomatous progression between untreated patients with normal-tension glaucoma and patients with therapeutically reduced intraocular pressures. Collaborative Normal-Tension Glaucoma Study Group. Am J Ophthalmol 1998;126:487-97.

5 Gordon MO, Beiser JA, Brandt JD, et al. The Ocular Hypertension Treatment Study: baseline factors that predict the onset of primary open-angle glaucoma. Arch Ophthalmol 2002;120:714-20; discussion 829-30.

6 The Advanced Glaucoma Intervention Study (AGIS): 7. The relationship between control of intraocular pressure and visual field deterioration. The AGIS Investigators. Am J Ophthalmol 2000;130:429-40.

7 Garway-Heath DF, Crabb DP, Bunce C, et al. Latanoprost for open-angle glaucoma (UKGTS): a randomised, multicentre, placebo-controlled trial. Lancet 2015:385:1295-304

8 Maier PC, Funk J, Schwarzer G, et al. Treatment of ocular hypertension and open angle glaucoma: meta-analysis of randomised controlled trials. BMJ 2005;331:134

9 King A, Azuara-Blanco A, Tuulonen A. Glaucoma. BMJ 2013:346:f3518.

10 National Institute of Health and Care Excellence (NICE). Glaucoma; Diagnosis and management of chronic open angle glaucoma and ocular hypertension CG85. London, UK: NICE, 2009

11 American Academy Ophthalmology. Primary Open Angle Glaucoma - Preferred Practise Patterns. Elsevier Inc, 2015.

12 European Glaucoma Society. Terminology and Guidelines for Glaucoma. 4th ed. Italy: Publicomm, 2014

13 Migdal C, Gregory W, Hitchings R. Long-term functional outcome after early surgery compared with laser and medicine in open-angle glaucoma. Ophthalmology 1994;101:1651-7.

14 Jay JL, Allan D. The benefit of early trabeculectomy versus conventional management in primary open angle glaucoma relative to severity of disease. Eye 1989;3(Pt 5):528-35.

15 Smith R. A comparison between medical and surgical treatment of glaucoma simplex-results of a prospective study. Trans Ophthalmol Soc Aust 1968;27:17-29.

16 Janz NK, Wren PA, Lichter PR, et al. The Collaborative Initial Glaucoma Treatment Study: interim quality of life findings after initial medical or surgical treatment of glaucoma. Ophthalmology 2001;108:1954-65.

17 Li T, Lindsley K, Rouse B, et al. Comparative effectiveness of first-line medications for primary open-angle glaucoma: a systematic review and network meta-analysis. Ophthalmology 2016;123:129-40.

18 Stalmans I, Gillis A, Lafaut AS, et al. Safe trabeculectomy technique: long term outcome. Br J Ophthalmol 2006;90:44-7.

19 Khaw PT, Chiang M, Shah P, et al. Enhanced trabeculectomy: the moorfields safer surgery system. Dev Ophthalmol 2012;50:1-28.

20 Cordeiro MF, Constable PH, Alexander RA, et al. Effect of varying the mitomycin-C treatment area in glaucoma filtration surgery in the rabbit. Invest Ophthalmol Vis SCi 1997;38:1639-46.

21 Zhou M, Wang W, Huang W, et al. Trabeculectomy with versus without releasable sutures for glaucoma: a meta-analysis of randomized controlled trials. BMC Ophthalmol 2014:14:41.

22 King AJ, Rotchford AP, Alwitry A, et al. Frequency of bleb manipulations after trabeculectomy surgery. Br J Ophthalmol 2007;91:873-7.

23 Kirwan JF, Lockwood AJ, Shah P, et al. Trabeculectomy in the 21st century: a multicenter analysis. Ophthalmology 2013;120:2532-9.

24 Thorpe $\mathrm{KE}$, Zwarenstein M, Oxman AD, et al. A pragmatic-explanatory continuum indicator summary (PRECIS): a tool to help trial designers. CMAJ 2009;180:E47-57.

25 Thorpe $\mathrm{KE}$, Zwarenstein M, Oxman AD, et al. A pragmatic-explanatory continuum indicator summary (PRECIS): a tool to help trial designers. J Clin Epidemiol 2009:62:464-75

26 Hodapp E, Parrish RK, Anderson DR. Clinical decisions in glaucoma. Mosby: St Louis C.V., 1993

27 Damji KF, Behki R, Wang L, et al. Canadian perspectives in glaucoma management: setting target intraocular pressure range. Can J Ophthalmol 2003:38:189-97.

28 Cairns JE. Trabeculectomy: Preliminary report of a new method. Am J Ophthalmol 1968;66:673-9. 
29 Hyman LG, Komaroff E, Heijl A, et al. Early Manifest Glaucoma Trial G. Treatment and vision-related quality of life in the early manifest glaucoma trial. Ophthalmology 2005; 112:1505-13.

30 Richman J, Lorenzana LL, Lankaranian D, et al. Relationships in glaucoma patients between standard vision tests, quality of life, and ability to perform daily activities. Ophthalmic Epidemiol 2010;17:144-51.

31 Guedes RA, Guedes VM, Freitas SM, et al. Quality of life of medically versus surgically treated glaucoma patients. J Glaucoma 2013;22:369-73.

32 Wu P, Xi S, Xia H, et al. Survey on vision-related quality of life and self-management among patients with glaucoma. J Glaucoma 2014;23:75-80.

33 EuroQol Group. EuroQol-a new facility for the measurement of health-related quality of life. Health Policy 1990;16:199-208.

34 Tosh J, Brazier J, Evans P, et al. A review of generic preference-based measures of health-related quality of life in visual disorders. Value Health 2012;15:118-27.

35 Burr JM, Kilonzo M, Vale L, et al. Developing a preference-based glaucoma utility index using a discrete choice experiment. Optom Vis Sci 2007;84:797-808.

36 Gutierrez P, Wilson MR, Johnson C, et al. Influence of glaucomatous visual field loss on health-related quality of life. Arch Ophthalmol 1997;115:777-84.

37 Jampel HD, Friedman DS, Quigley $H$, et al. Correlation of the binocular visual field with patient assessment of vision. Invest Ophthalmol Vis Sci 2002;43:1059-67.

38 Nelson P, Aspinall P, Papasouliotis 0, et al. Quality of life in glaucoma and its relationship with visual function. J Glaucoma 2003;12:139-50.

39 Health Utilities Inc. Multi-Attribute Health Status Classification System: Health Utilities Index Mark 3 (HUI3). http://www.healthutilities.com/hui3.htm.

40 Devlin N, Shah K, Feng Y, et al. Valuing Health-Related Quality of Life: an EQ5D-5L Value Set for England, Research Paper 16/01. London: Office of Health Economic, 2016.

41 Burr JM, Botello-Pinzon P, Takwoingi Y, et al. Surveillance for ocular hypertension: an evidence synthesis and economic evaluation. Health Technol Assess 2012;16:1-271.

42 Burr JM, Campbell MK, Campbell SE, et al. Developing the clinical components of a complex intervention for a glaucoma screening trial: a mixed methods study. BMC Med Res Methodol 2011;11:54.

43 Hernández RA, Burr JM, Vale LD. Economic evaluation of screening for open-angle glaucoma. Int J Technol Assess Health Care 2008;24:203-11.

44 Prior M, Ramsay CR, Burr JM,et al. Theoretical and empirical dimensions of the Aberdeen Glaucoma Questionnaire: a cross sectional survey and principal component analysis. BMC Ophthalmol 2013;13:72.

45 Chehamzeh J. Assessment of glaucoma: Using patient reported measures in randomised controlled trials. University of Aberdeen,2011.

46 Suñer IJ, Kokame GT, Yu E, et al. Responsiveness of NEI VFQ-25 to changes in visual acuity in neovascular AMD: validation studies from two phase 3 clinical trials. Invest Ophthalmol Vis Sci 2009;50:3629-35.

47 Musch DC, Gillespie BW, Lichter PR, et al. Visual field progression in the collaborative initial glaucoma treatment study the impact of treatment and other baseline factors. Ophthalmology 2009;116:200-7.

48 Bengtsson B, Patella VM, Heijl A. Prediction of glaucomatous visual field loss by extrapolation of linear trends. Arch Ophthalmol 2009;127:1610-5.

49 McNaught Al, Crabb DP, Fitzke FW, et al. Modelling series of visual fields to detect progression in normal-tension glaucoma. Graefes Arch Clin Exp Ophthalmol 1995;233:750-5.

50 Medeiros FA, Gracitelli CP, Boer ER, et al. Longitudinal changes in quality of life and rates of progressive visual field loss in glaucoma patients. Ophthalmology 2015;122:293-301.
51 Carpenter J, Kenward M. Missing data in clinical trials - a practical guide. Birmingham: NIfH, 2008.

52 White IR, Horton NJ, Carpenter J, et al. Strategy for intention to treat analysis in randomised trials with missing outcome data. BMJ 2011;342:d40.

53 Emsley R, Dunn G, White IR. Mediation and moderation of treatment effects in randomised controlled trials of complex interventions. Stat Methods Med Res 2010;19:237-70

54 Stead R, Azuara-Blanco A, King AJ. Attitudes of consultant ophthalmologists in the UK to initial management of glaucoma patients presenting with severe visual field loss: a national survey. Clin Exp Ophthalmol 2011;39:858-64.

55 McKean-Cowdin R, Wang Y, Wu J, et al. Impact of visual field loss on health-related quality of life in glaucoma: the Los Angeles Latino Eye Study. Ophthalmology 2008;115:941-8.

56 Ramulu P. Glaucoma and disability: which tasks are affected, and at what stage of disease? Curr Opin Ophthalmol 2009;20:92-8.

57 Ramulu PY, Swenor BK, Jefferys JL, et al. Difficulty with out-loud and silent reading in glaucoma. Invest Ophthalmo/ Vis Sci 2013;54:666-72.

58 Ramulu PY, van Landingham SW, Massof RW, et al. Fear of falling and visual field loss from glaucoma. Ophthalmology 2012;119:1352-8.

59 Ramulu PY, West SK, Munoz B, et al. Driving cessation and driving limitation in glaucoma: the salisbury eye evaluation project. Ophthalmology 2009;116:1846-53.

60 Ramulu PY, West SK, Munoz B, et al. Glaucoma and reading speed: the salisbury eye evaluation project. Arch Ophthalmol 2009;127:82-7.

61 Haymes SA, Leblanc RP, Nicolela MT, et al. Risk of falls and motor vehicle collisions in glaucoma. Invest Ophthalmo/ Vis Sci 2007;48:1149-55.

62 Haymes SA, LeBlanc RP, Nicolela MT, et al. Glaucoma and on-road driving performance. Invest Ophthalmol Vis Sci 2008:49:3035-41.

63 Goldberg I, Clement $\mathrm{Cl}$, Chiang TH, et al. Assessing quality of life in patients with glaucoma using the Glaucoma Quality of Life-15 (GQL-15) questionnaire. J Glaucoma 2009;18:6-12.

64 Owsley C, McGwin G. Vision and driving. Vision Res 2010;50:2348-61.

65 Musch DC, Gillespie BW, Niziol LM, et al. Intraocular pressure control and long-term visual field loss in the Collaborative Initial Glaucoma Treatment Study. Ophthalmology 2011:118:1766-73.

66 Musch DC, Gillespie BW, Palmberg PF, et al. Visual field improvement in the collaborative initial glaucoma treatment study. Am J Ophthalmol 2014; 158:96-104

67 Musch DC, Gillespie BW, Niziol LM, et al. Cataract extraction in the collaborative initial glaucoma treatment study: incidence, risk factors, and the effect of cataract progression and extraction on clinical and quality-of-life outcomes. Arch Ophthalmol 2006;124:1694-700.

68 Rahman MQ, Beard SM, Discombe R, et al. Direct healthcare costs of glaucoma treatment. Br J Ophthalmol 2013;97:720-4.

69 Ang GS, Eke T. Lifetime visual prognosis for patients with primary open-angle glaucoma. Eye 2007;21:604-8

70 Grødum K, Heijl A, Bengtsson B. Glaucoma and mortality. Graefes Arch Clin Exp Ophthalmol 2004;242:397-401.

71 Goh YW, Ang GS, Azuara-Blanco A. Lifetime visual prognosis of patients with glaucoma. Clin Exp Ophthalmol 2011;39:766-70.

72 Peters $D$, Bengtsson $B$, Heijl A. Lifetime risk of blindness in open-angle glaucoma. Am J Ophthalmol 2013;156:724-30. 